\title{
Elimination of negative differential conductance in an asymmetric molecular transistor by an ac-voltage
}

\author{
Bing Dong and X.L. Lei \\ Department of Physics, Shanghai Jiaotong University, \\ 1954 Huashan Road, Shanghai 200030, China \\ N. J. M. Horing \\ Department of Physics and Engineering Physics, \\ Stevens Institute of Technology, Hoboken, New Jersey 07030, USA
}

(Dated: October 26, 2018)

\begin{abstract}
We analyze resonant tunneling subject to a non-adiabatic time-dependent bias-voltage through an asymmetric single molecular quantum dot with coupling between the electronic and vibrational degrees of freedom using a Tien-Gordon-type rate equation. Our results clearly exhibit the appearance of photon-assisted satellites in the current-voltage characteristics and the elimination of hot-phonon-induced negative differential conductance with increasing ac driving amplitude for an asymmetric system. This can be ascribed to an ac-induced suppression of unequilibrated (hot) phonons in an asymmetric system.

PACS numbers: 85.65.+h, 71.38.-k, 72.40.+w, 73.63.Kv
\end{abstract}


Recently, phonon-mediated de tunneling through a quantum dot (QD) with coupling to an internal vibrational (phonon) mode (IVM) has attracted much interest, both experimental $1,2, \underline{3}$ and theoretical. $, \frac{4}{,}, 6,7,8,9,10,11$ Electronic transport measurements in suspended Carbon nanotubes (CNT) indicate that the current-voltage characteristics display perfect signatures of phonon-mediated tunneling, e.g. stepwise structures having equal widths in voltage and gradual height reduction by the Franck-Condon (FC) factor $\stackrel{2,3}{*}$ Furthermore, striking negative differential conductance (NDC) and current peaks have been observed at the onsets of phonon steps, $\frac{3}{3}$ which are ascribed to the combination of highly asymmetric tunnel-coupling rates and the voltage-triggered unequilibrated (hot) phonon effect. ${ }^{9}, 10,11$ On the other hand, ac-transport through a single molecule has also attracted considerable interest, but without consideration of electron-phonon coupling (EPC) $\stackrel{12}{=}$ In this letter, we will examine ac-transport in the presence of EPC when a time-dependent biasvoltage is applied between the two electrodes in the non-adiabatic regime $\underline{13}^{\text {We find that }}$ application of a strong ac-amplitude causes suppression of hot phonons and thus elimination of NDC for a large asymmetric system.

We consider a quantum dot (QD) with one spinless level coupled to two electrodes, and also linearly coupled to a molecular IVM. The model Hamiltonian is

$$
H=H_{\text {leads }}+H_{m o l}+H_{B}+H_{T}
$$

with

$$
\begin{aligned}
H_{\text {leads }} & =\sum_{\eta, \mathbf{k}} \varepsilon_{\eta \mathbf{k}} c_{\eta \mathbf{k}}^{\dagger} c_{\eta \mathbf{k}}, \\
H_{m o l} & =\varepsilon_{d} c_{d}^{\dagger} c_{d}+\omega_{0} a^{\dagger} a+\lambda c_{d}^{\dagger} c_{d}\left(a^{\dagger}+a\right), \\
H_{T} & =\sum_{\eta, \mathbf{k}}\left(V_{\eta} c_{\eta \mathbf{k}}^{\dagger} c_{d}+\text { H.c. }\right)
\end{aligned}
$$

where $c_{\eta \mathbf{k}}^{\dagger}\left(c_{\eta \mathbf{k}}\right)$ is the creation (annihilation) operator of an electron with momentum $\mathbf{k}$ in lead $\eta(\eta=L, R)$. The energies $\varepsilon_{\eta \mathbf{k}}(t)=\varepsilon_{\eta \mathbf{k}}^{0}+e U_{\eta}(t)$ include rigid shifts of the Fermi energies of the electrons in the leads due to the applied time-dependent bias-voltage, $U_{\eta}(t)=$ $U_{\eta}^{0}+u_{\eta} \cos (\Omega t)$, with $U_{\eta}^{0}\left(u_{\eta}\right)$ being the $\mathrm{dc}(\mathrm{ac})$ part of bias-voltage and $\Omega$ is the driving frequency. $c_{d}^{\dagger}\left(c_{d}\right)$ is the creation (annihilation) operator for a spinless electron in the QD. $a^{\dagger}(a)$ is the phonon creation (annihilation) operator for the IVM with energy $\omega_{0} \cdot \lambda$ represents the EPC constant; $V_{\eta}$ describes the tunnel-coupling between the QD and lead $\eta$. Here, we 
assume that the Fermi energies of two leads are zero at equilibrium and $U_{L}^{0}=-U_{R}^{0}=e V / 2$, $u_{L}=-u_{R}=e V_{a c}$ ( $V$ and $V_{a c}$ are the dc bias-voltage and ac-bias amplitudes, respectively). In the following, we will use units where $\hbar=k_{B}=e=1$.

It is well-known that the electron-phonon interaction term in Eq. (1c) can be eliminated by a canonical transformation,,$\frac{14}{1}$ leading to a renormalization of the parameters, $\widetilde{\varepsilon}_{d}=\varepsilon_{d}-g \lambda$ $\left(g=\lambda / \omega_{0}\right)$, and of the tunnel-coupling, $V_{\eta} \exp \left[g\left(a^{\dagger}+a\right)\right]$. In the weak tunneling regime and high temperature approximation, $\Gamma_{\eta} \ll T, \omega_{0}$ ( $\Gamma_{\eta}$ is the tunneling rate of lead $\eta$ and $T$ is the temperature), rate equations for the electron-phonon joint probabilities, $\rho_{00}^{n}$ and $\rho_{11}^{n}$ for zero- and one-electron together with $n$ excited phonons on the molecule (incorporated with the FC-modified tunneling rates) are physically appropriate for the description of resonant

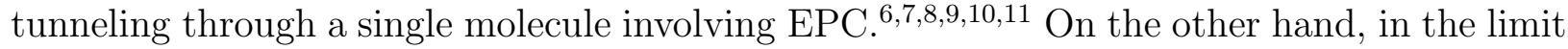
of high driving frequency, $\Omega \gg \Gamma, T$, of interest in this letter, the ac-bias oscillates so fast that an electron experiences many cycles of the ac-bias during its presence inside the dot, and thus can not sense the details of the dynamics within a single period. Correspondingly, the rate equations can be established by directly applying a Tien-Gordon-type tunneling rate in the presence of such an ac-bias in the non-adiabatic limit, $, 15,16$

$$
\begin{aligned}
& \dot{\rho}_{00}^{n}=\sum_{m}\left(\Gamma_{n m}^{-} \rho_{11}^{m}-\Gamma_{n m}^{+} \rho_{00}^{n}\right), \\
& \dot{\rho}_{11}^{n}=\sum_{m}\left(\Gamma_{m n}^{+} \rho_{00}^{m}-\Gamma_{m n}^{-} \rho_{11}^{n}\right),
\end{aligned}
$$

with the normalization relation $\sum_{n}\left(\rho_{00}^{n}+\rho_{11}^{n}\right)=1$. The electronic tunneling rates are defined as

$$
\begin{aligned}
\Gamma_{n m}^{+}= & \sum_{\eta} \Gamma_{\eta, n m}^{+}=\sum_{\eta} \Gamma_{\eta} \gamma_{n m} \sum_{j=-\infty}^{\infty}\left[J_{j}\left(\frac{u_{\eta}}{\Omega}\right)\right]^{2} \\
& \times f_{\eta}\left(\widetilde{\epsilon}_{d}+(m-n) \omega_{0}-j \Omega\right), \\
\Gamma_{n m}^{-}= & \sum_{\eta} \Gamma_{\eta, n m}^{-}=\sum_{\eta} \Gamma_{\eta} \gamma_{n m} \sum_{j=-\infty}^{\infty}\left[J_{j}\left(\frac{u_{\eta}}{\Omega}\right)\right]^{2} \\
& \times\left[1-f_{\eta}\left(\widetilde{\epsilon}_{d}+(m-n) \omega_{0}-j \Omega\right)\right],
\end{aligned}
$$

with the $F C$ factor given by $(p=\min \{m, n\}$ and $q=\max \{m, n\}$, denoting the smaller and larger of the quantities $m$ and $n$, respectively)

$$
\gamma_{n m}=e^{-g^{2}} g^{2|m-n|} \frac{p !}{q !}\left[L_{p}^{|m-n|}\left(g^{2}\right)\right]^{2}
$$


where $L_{n}^{m}(x)$ is the generalized Laguerre polynomial. $J_{j}(x)$ is the Bessel function of order j. $f_{\eta}(\epsilon)=\left[1+e^{\left(\epsilon-\mu_{\eta}\right) / T}\right]^{-1}$ is the Fermi-distribution function. Moreover, the dc current can be calculated by solving the rate equations, Eqs. (2) and (3), in the steady state condition and evaluating the net tunneling rate through one of electrodes (for example, the left lead):

$$
I=\sum_{n m}\left(\Gamma_{L, n m}^{+} \rho_{00}^{n}-\Gamma_{L, m n}^{-} \rho_{11}^{n}\right) .
$$

Note that if the driving amplitude vanishes, $u_{\eta}=0$, the above equations (2)-(7) reduce exactly to the rate equations formulated without an ac-bias. $\underline{6}, \underline{7}, \underline{9}, \underline{10}, 10.11$

In our numerical calculation, we set the parameters as: $\omega_{0}=1$ is the energy unit, $\Gamma_{R} / \Gamma_{L}=10^{3}, g=1, \widetilde{\varepsilon}_{d}=0, T=0.02 \omega_{0}$, and the driving frequency is $\Omega=0.5 \omega_{0}$. Note that we choose a large asymmetry in the tunneling rates, $\Gamma_{R} / \Gamma_{L}=10^{3}$, and an intermediate EPC strength, $g$. The transport properties of this specific system exhibit weak NDC at the onsets of phonon steps under hot phonon condition. $\stackrel{10,11}{=}$ In particular, numerical fits of the experimentally measured data for the $I-V$ curves show $g \simeq 1$ for long CNTs. $\frac{3}{}$

Figure 1 shows the dc current-bias-voltage characteristics as a function of various driving amplitudes. For comparison, we also plot the results without an ac-bias, $V_{a c}=0$, as the solid line, showing (1) step behavior with width equal to $2 \omega_{0}$, (2) the FC factor-suppressed height, and (3) NDC at the onsets of phonon steps starting from the second step. When an ac-bias is applied, it is obvious to see from Fig. 1 that a photon-assisted tunneling featureconstituted of new steps at $V=2 n \Omega$ ( $n$ is integer) - is superimposed on the phonon-mediated $I-V$ characteristics. For stronger driving amplitudes, the suppression of current is more pronounced in the weak dc-bias region, $V<2 \Omega$. However, in the strong dc-bias region, $V>4 \omega_{0}$, the application of the ac-bias always enhances the current. Such nonuniform behavior is reflected in the structure of the Bessel function in the tunneling rates, Eqs. (4) and (5). More interestingly, we observe that for a weak ac-amplitude, $V_{a c}=0.2 \omega_{0}$, the position of NDC moves to the ends of the phonon steps, while in the cases of strong acamplitudes, $V_{a c}=0.5 \omega_{0}$ and $1.0 \omega_{0}$, the NDC even disappears. It should be noted that strong vibrational relaxation can result in the elimination of NDC even in the absence of an acbias $\stackrel{10,11}{1}$ In that case, photon-assisted steps still remain in the current-voltage characteristics when an ac-bias is applied (not shown here) $\underline{\underline{13}}$

To gain insight into this peculiar behavior, we adopt the method of Ref. 10 by examining the phonon occupation numbers (PONs), $\rho_{00}^{n}$. For the positive dc bias-voltage range of 
interest in this letter, $V>0$, the QD is always in an empty occupation state owing to $\Gamma_{R} \gg \Gamma_{L}$ (so that the electron has a much stronger tunneling-out rate to the right lead than the tunneling-in rate from the left lead). Therefore, a nonzero value of $\rho_{00}^{n}$ indicates an open channel contributing to the current. In this case, we can rewrite the current formula, Eq. (17), with good accuracy, as

$$
I=\sum_{n}^{N} \mathcal{I}_{n} \rho_{00}^{n},
$$

with ( $N$ denotes the maximum number of PONs of the open channels)

$$
\begin{gathered}
\mathcal{I}_{n}=\sum_{m} \Gamma_{L, n m}^{+}=\Gamma_{L} \sum_{m} \gamma_{n m} \sum_{j=-\infty}^{\infty}\left[J_{j}\left(\frac{u_{\eta}}{\Omega}\right)\right]^{2} \\
\times \Theta\left(-\widetilde{\epsilon}_{d}-(m-n) \omega_{0}+j \Omega+V / 2\right)
\end{gathered}
$$

$[\Theta(x)$ is the Heaviside step function]. Starting from these two equations, we interpret the NDC and positive differential conductance (PDC) behaviors of the $I-V$ characteristics in the presence of the ac-bias in Fig. 1.

Figure 2 displays the $\rho_{00}^{n}$ under several dc-bias voltages and ac-amplitudes as well. It is evident that for small dc-bias, $V_{1}=2.0 \omega_{0}$ (the panels of the first row in Fig. 2), only the channel with the PON $n=0$ is active, $\rho_{00}^{0} \approx 1$, and the application of an ac-bias has very little influence on the PON. In these situations, we have $N=0$ and thus

$$
I_{1} \simeq \Gamma_{L} \sum_{m} \gamma_{0 m} \mathcal{J}_{m}
$$

with $\mathcal{J}_{m}=\sum_{j=2(m-1)}^{\infty}\left[J_{j}\left(\frac{V_{a c}}{\Omega}\right)\right]^{2}$. If the ac-bias vanishes, the current becomes (we use $\bar{I}$ here to denote the current in the absence of an ac-bias)

$$
\bar{I}_{1} \simeq \Gamma_{L}\left(\gamma_{00}+\gamma_{01}\right)
$$

When the dc-bias exceeds the transition point $V_{1}=2.0 \omega_{0}$, for example $V_{2}=2.3 \omega_{0}$ (the panels in the second row of Fig. 2), two channels, $n=0$ and 1, are open in the absence of an ac-bias, which indicates an unequilibrated (hot) phonon distribution. Correspondingly, the current is

$$
\begin{aligned}
\bar{I}_{2} & \simeq \Gamma_{L}\left[\rho_{00}^{0}\left(\gamma_{00}+\gamma_{01}\right)+\rho_{10}^{1}\left(\gamma_{10}+\gamma_{11}+\gamma_{12}\right)\right] \\
& \simeq \Gamma_{L} \gamma_{00}+\Gamma_{L} \rho_{00}^{1}\left(\gamma_{11}+\gamma_{12}-\gamma_{00}\right)
\end{aligned}
$$


because $\rho_{00}^{0}+\rho_{00}^{1} \simeq 1$. As a result, $\bar{I}_{2}-\bar{I}_{1} \simeq \Gamma_{L} \rho_{00}^{1} e^{-g^{2}} \rho_{00}^{1} g^{4}\left(g^{2} / 2-1\right)<0$ due to $g=1$ in this letter, leading to the appearance of NDC. 10 When an ac-bias is applied, we findstrikingly-from Fig. 2 that the hot phonon is suppressed and the $n=1$ channel becomes closed. This suppression of $\rho_{00}^{1}$ stems from a weak increase of $\rho_{11}^{n}$ due to photon-assisted processes. As a result, we have $I_{2} \approx I_{1}$ and the NDC reverts to a pure PDC at the onsets of the phonon steps. Furthermore, for $V_{3}=3.0 \omega_{0}$ and $V_{4}=3.3 \omega_{0}$, two channels, $n=0$ and 1 , are activated by the dc-bias and we readily obtain $\bar{I}_{3} \simeq \bar{I}_{4}$. The ac-bias-induced suppression of hot phonons changes the current of the case of $V_{3}$ as $I_{3} \simeq I_{1}$. However, the application of a weak ac-amplitude, $V_{a c}=0.2 \omega_{0}$, can not completely suppress the $n=1$ hot phonon at the moderately stronger dc-bias $V_{4}$, and thus

$$
\begin{aligned}
I_{4} & \simeq \Gamma_{L} \rho_{00}^{0} \sum_{m} \gamma_{0 m} \mathcal{J}_{m}+\Gamma_{L} \rho_{00}^{1} \sum_{m} \gamma_{1 m} \mathcal{J}_{m}^{\prime} \\
& =I_{3}+\Gamma_{L} \rho_{00}^{1} \sum_{m}\left(\gamma_{1 m} \mathcal{J}_{m}^{\prime}-\gamma_{0 m} \mathcal{J}_{m}\right)
\end{aligned}
$$

with $\mathcal{J}_{m}^{\prime}=\sum_{j=2 m-5}^{\infty}\left[J_{j}\left(\frac{V_{a c}}{\Omega}\right)\right]^{2}$. The second term of the last line of Eq. (13) may be positive or negative depending on the EPC constant $g$ and the ac-amplitude $V_{a c}$. For the weak ac-amplitude $V_{a c}=0.2 \omega_{0}$, it is negative, leading to NDC; while for stronger amplitudes, $V_{a c}=0.5 \omega_{0}$ and $1.0 \omega_{0}$, it is positive and PDC is observed.

In summary, we have employed the Tien-Gordon-type rate equations incorporated with the FC model to analyze photon-assisted resonant tunneling through an asymmetric single molecular QD with EPC in the non-adiabatic and high frequency regime. Our results predict that the application of an ac-bias causes suppression of the dc-bias-triggered hot phonon, leading to the elimination of NDC in the $I-V$ characteristics.

This work was supported by Projects of the National Science Foundation of China, the Shanghai Municipal Commission of Science and Technology, the Shanghai Pujiang Program, and Program for New Century Excellent Talents in University (NCET). 
1 H. Park, J. Park, A. Lim, E. Anderson, A. Alivisatos, and P. McEuen, Nature 407, 57 (2000).

2 B.J. LeRoy, S.G. Lemay, J. Kong, and C. Dekker, Nature 432, 371 (2004); B.J. LeRoy, J. Kong, V.K. Pahilwani, C. Dekker, and S.G. Lemay, Phys. Rev. B 72, 75413 (2005).

3 S. Sapmaz, P. Jarillo-Herrero, Ya.M. Blanter, C. Dekker, and H.S.J. van der Zant, Phys. Rev. Lett. 96, 26801 (2006); S. Sapmaz, P. Jarillo-Herrero, Ya.M. Blanter, and H.S.J. van der Zant, New J. Phys. 7, 243 (2005).

4 D. Boese and H. Schoeller, Europhys. Lett. 54, 668 (2001).

5 A.S. Alexandrov and A.M. Bratkovsky, Phys. Rev. B 67, 235312 (2003); K.D. McCarthy, N. Prokof'ev, and M.T. Tuominen, Phys. Rev. B 67, 245415 (2003).

6 A. Mitra, I. Aleiner, and A.J. Millis, Phys. Rev. B 69, 245302 (2004).

7 J. Koch and F. von Oppen, Phys. Rev. Lett. 94, 206804 (2005); J. Koch, M.E. Raikh, and F. von Oppen, Phys. Rev. Lett. 95, 56801 (2005).

8 K.C. Nowack and M.R. Wegewijs, cond-mat/0506552 (2005); M.R. Wegewijs, K.C. Nowack, New J. Phys. 7, 239 (2005).

9 J. Koch and F. von Oppen, Phys. Rev. B 72, 113308 (2005).

10 A. Zazunov, D. Feinberg, and T. Martin, Phys. Rev. B 73, 115405 (2006).

11 X. Y. Shen, Bing Dong, X.L. Lei, and N.J.M. Horing, unpublished.

12 S. Camalet, S. Kohler, and P. Hänggi, Phys. Rev. B 70, 155326 (2004); P. Hänggi, S. Kohler, J. Lehmann, and M. Strass, Phys. Rep. 406, 379 (2005); P. Hänggi, S. Kohler, J. Lehmann, and M. Strass, Lect. Notes Phys. 680, 55 (2005).

13 Bing Dong, H.L. Cui, and X.L. Lei, Phys. Rev. B 69, 205315 (2004).

14 G.D. Mahan, Many-Particle Physics. (Third edition, Kluwer Academic/Plenum Publisher, New York, 2000).

15 P.K. Tien and J.R. Gordon, Phys. Rev. 129, 647 (1963); C. Bruder and H. Schoeller, Phys. Rev. Lett. 72, 1076 (1994).

16 W.G. van der Wiel, T.H. Oosterkamp, S. De Franceschi, C.J.P.M. Harmans, L.P. Kouwenhoven, cond-mat/9904359. 


\section{Figure Caption}

FIG.1: $I$ - $V$ curves as functions of ac bias-voltage amplitudes, $V_{a c} / \omega_{0}=0,0.2,0.5$, and 1.0, for a fixed driving frequency, $\Omega / \omega_{0}=0.5$. The parameters we use in the calculation are: $g=1, \Gamma_{L} / \Gamma_{R}=10^{-3}$, and $T / \omega_{0}=0.02$.

FIG.2: Phonon probability distributions for increasing ac-amplitude, $V_{a c}$, under different dc bias-voltages, $V / \omega_{0}=2.0,2.3,3.0$, and 3.3. Other parameters are the same as in Fig. 1. 


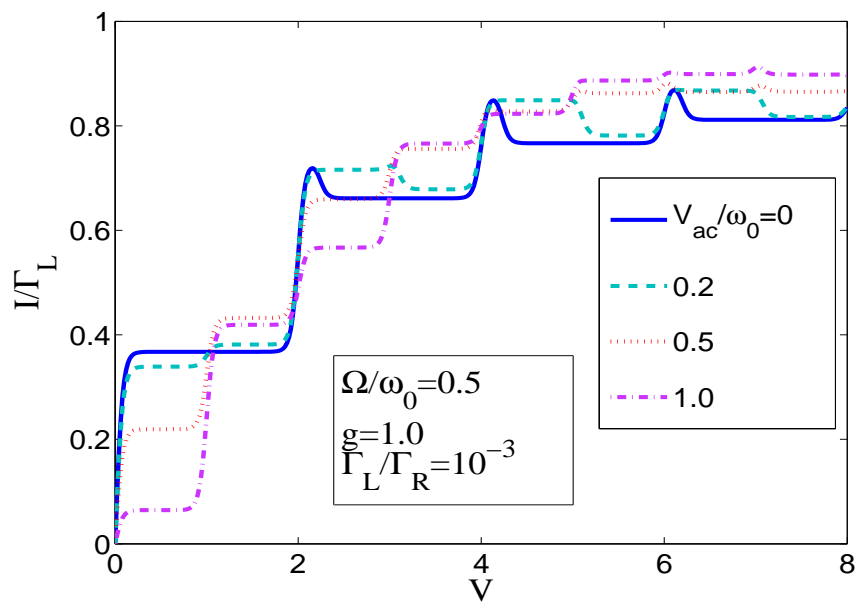

Figure 1: $I-V$ curves as functions of ac bias-voltage amplitudes, $V_{a c} / \omega_{0}=0,0.2,0.5$, and 1.0, for a fixed driving frequency, $\Omega / \omega_{0}=0.5$. The parameters we use in the calculation are: $g=1$, $\Gamma_{L} / \Gamma_{R}=10^{-3}$, and $T / \omega_{0}=0.02$. 

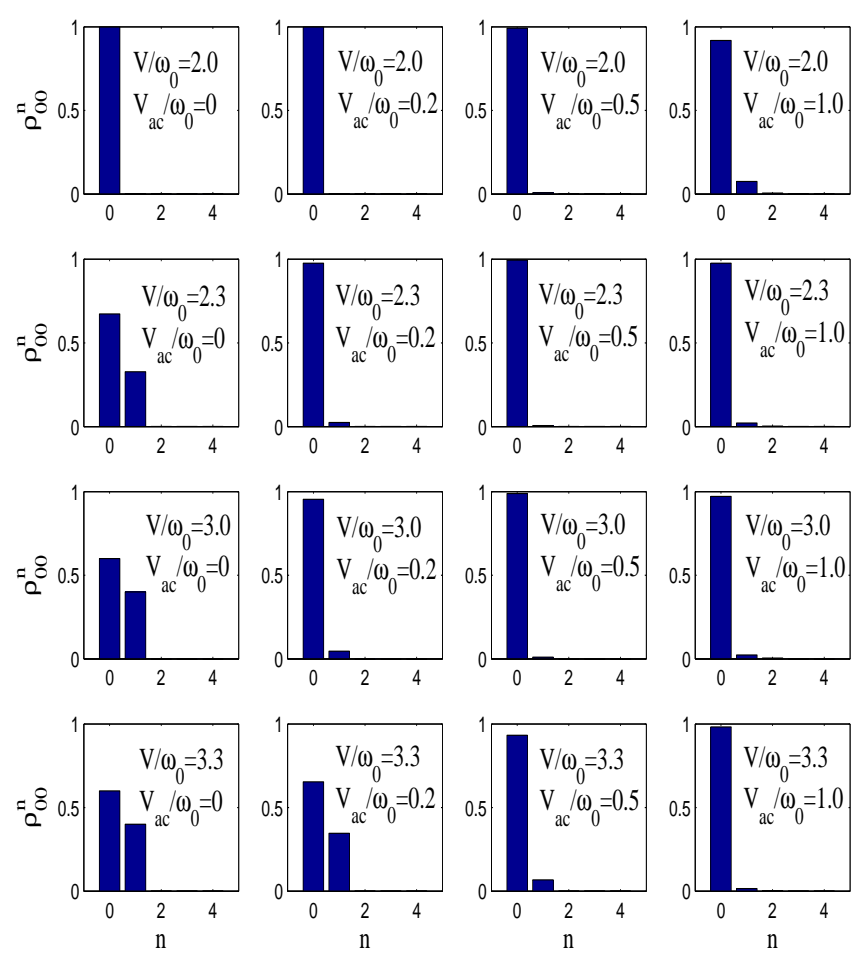

Figure 2: Phonon probability distributions for increasing ac-amplitude, $V_{a c}$, under different dc bias-voltages, $V / \omega_{0}=2.0,2.3,3.0$, and 3.3. Other parameters are the same as in Fig. 1. 